Questions de communication

$24 \mid 2013$

Renouvellement des mises en scène télévisuelles de la politique

\title{
Aurélie DUDEZERT, La connaissance dans les entreprises
}

Paris, Éd. La Découverte, coll. Repères, 2013, 128 pages

\section{Alexandre Eyries}

\section{OpenEdition}

\section{Journals}

Édition électronique

URL : http://journals.openedition.org/questionsdecommunication/8794

DOI : 10.4000/questionsdecommunication.8794

ISSN : 2259-8901

\section{Éditeur}

Presses universitaires de Lorraine

Édition imprimée

Date de publication : 31 décembre 2013

Pagination : 278-280

ISBN : 978-2-8143-0182-5

ISSN : 1633-5961

\section{Référence électronique}

Alexandre Eyries, "Aurélie dudezert, La connaissance dans les entreprises », Questions de communication

[En ligne], 24 | 2013, mis en ligne le 01 février 2014, consulté le 22 septembre 2020. URL : http://

journals.openedition.org/questionsdecommunication/8794; DOI : https://doi.org/10.4000/

questionsdecommunication.8794 
Des inconsistances du rapport de police (rapport Henri Chavin, 194I) à l'impossibilité de prouver que Jean Coutrot avait été à la tête d'une entente secrète réunissant des technocrates désireux d'infiltrer le pouvoir politique, rien ne fait barrage aux discours antisynarchiques qui jouissent d'une belle santé depuis la Libération (cinquième chapitre, pp. 167-217). À partir de cette époque, la théorie se délocalise plus encore. Les sociétés secrètes du fascisme français (avec une nouvelle mise en cause de Jean Coutrot) et l'infiltration cléricale (les jésuites toujours en ligne de mire des antisynarchistes de gauche comme de droite) sont pointées du doigt. Le communisme (pp. 172-176), d'abord, dans les colonnes d'Action, découvre dans la synarchie l'ennemi par excellence, qui s'oppose à ses intérêts et à ceux de la France (le Vatican, les réactionnaires anglo-saxons, les socialistes, Charles de Gaulle).

Les forces synarchiques continuent donc à opérer - la commission Trilatérale fut une de leurs expressions et fut attaquée par un antimondialisme très présent sur l'échiquier politique. Et nombre des épisodes fondateurs furent réactualisés, sans grande originalité, pour confirmer la réalité de son influence décisive au sein des hautes sphères du pouvoir. L'extrême droite la brandit avec des auteurs comme Henry Coston et Louis Daménie - dans ce dernier cas, il était nouvellement fait référence à lean Coutrot (pp. 192193), tandis que la gauche anticapitaliste s'en empare pour expliquer les événements politiques dominants - nouvelle apparition de lean Coutrot-pour expliquer l'influence de la grande banque internationale dans la désignation d'importantes autorités politiques dans des pays européens (pp. 219-220).

La minutieuse reconstruction d'Olivier Dard met en évidence les difficultés rencontrées à l'heure du bilan pour les sphères du pouvoir (p. 225). La synarchie constitue une simplification politique (comme instrument de déqualification), mais également intellectuelle - grille de lecture qui, comme il ressort du texte, n'a pas été sans intérêt dans la littérature académique (pp. 212, 216-217). Le recours à cet instrument semble également être dépendant de conditions externes, les périodes de crise étant les plus favorables (pp. 133, | 4| - | 42, 219). Ayant accumulé des décennies d'évolution de la culture technocratique dont les limites se sont vérifiées, le contexte actuel ne rend pas nos sociétés imperméables à la prolifération de ces manifestations de mythologie politique. L'auteur n'est pas persuadé qu'elles ne puissent ressurgir au sein de sociétés déboussolées par le spectacle de limpotence des élites politiques et par le consensus sur des décisions difficilement acceptées socialement. Ainsi « le présent serait[-t-il] un vivier enrichissant et le passé un réservoir inépuisable de références 》 (p. 232). Loin de réduire la compréhension du phénomène aux interminables « fables » des protagonistes, Olivier Dard présente la synarchie comme une représentation enracinée, c'est-à-dire comme un phénomène de croyance, producteur de sens (pp. 125, 127, 177). Sa conclusion (pp. 219-232) approfondit cette clé de lecture qui, intégrant les récits rapportés, les déborde amplement pour atteindre des dérivations problématiques d'une rare profondeur... et d'une rare contemporanéité.

\section{Humberto Cucchetti \\ CEIL, Conseil national de recherches scientifiques et techniques, AR-CI 083ACA hcucchetti@ceil-conicet.gov.ar}

\section{Aurélie DudEZERT, La connaissance dans les entreprises.} Paris, Éd. La Découverte, coll. Repères, 2013, I28 p.

Le livre d'Aurélie Dudezert, mâtre de conférences habilitée à diriger les recherches en sciences de gestion à l'École centrale de Paris et responsable de l'équipe de recherche Epocc (Équipe de recherche en économie et gestion sur les politiques de croissances fondées sur la connaissance) - lève le voile sur un aspect peu connu mais pourtant d'une importance capitale dans la vie d'une organisation (qu'il s'agisse d'une administration ou d'une institution) : la gestion des connaissances sous toutes leurs formes, connaissances qui excèdent d'ailleurs largement les frontières du droit de la propriété intellectuelle et industrielle. Dans le monde économique actuel, la création de valeur découle en majeure partie de l'exploitation de la connaissance de l'entreprise et non plus, comme autrefois, de l'exploitation et de la transformation de ressources naturelles. Dans cet environnement hyperconcurrentiel, les connaissances de l'entreprise (produites, transmises ou capitalisées par elle) sont une ressource capitale à protéger pour conquérir un net avantage sur les concurrents directs du même secteur de marché.

Cependant, la connaissance n'est pas une ressource tout à fait comme les autres. L'un des mérites de l'ouvrage d'Aurélie Dudezert est de donner des clés de compréhension des modalités managériales émergentes spécifiques à la gestion de la connaissance, les nouveaux enjeux induits par celle-ci ainsi que les nouvelles formes d'entreprise et d'action collective qui sont esquissées. Le livre structuré en trois chapitres - respectivement intitulés « La connaissance organisationnelle: gérer l'ingérable »(pp. 9-38), 
« Le système de gestion des connaissances comme mode de gestion "classique" de la connaissance ») (pp. 39-68) et « Réinventer l'organisation : le modèle de l'entreprise centrée connaissance » (pp. 69-98) présente de manière synthétique et rigoureuse les évolutions de la gestion des connaissances, les resitue dans l'histoire des approches et des pratiques du management et tente d'en évaluer les conséquences sur le plan socioéconomique.

Dans l'introduction (pp. 3-8), l'auteure rappelle que le monde est entré, depuis la fin des années 90, dans une économie dite de la connaissance qui se caractérise par le fait que la part des emplois intensifs de ce secteur s'est considérablement accrue, le poids économique des secteurs d'information est devenu déterminant et la part du capital intangible a dépassé celle du capital tangible. Dans cette économie contemporaine, « la connaissance est un actif déterminant sur le marché concurrentiel et une ressource clef pour les entreprises » (p. 3). D'abord, la production de connaissances devient un enjeu pour construire une nouvelle politique industrielle capable de lutter contre la désindustrialisation touchant de nombreux pays dans le monde (notamment occidentaux). C'est ainsi que, selon la chercheuse, « les organisations traditionnellement créatrices de connaissances comme les centres de recherche, les universités, les établissements d'enseignement supérieur deviennent les acteurs clefs de cette transformation industrielle. Elles sont invitées non seulement à confirmer et accrôtre leur niveau de production de connaissances, mais également à travailler leur transférabilité vers le monde économique » (pp. 3-4). Cela conduit naturellement les entreprises à prendre progressivement conscience de l'évolution économique notable faisant de la connaissance une ressource cruciale pour assurer la compétitivité de l'entreprise sur les plans national et international ainsi que pour garantir la qualité constante des services qu'elle offre à ses clients. «Dans tous ses domaines de compétence (recherche et développement ou $R \& D$, production, marketing, comptabilité, finance, etc.), [les compétences de l'entreprise] deviennent une ressource à gérer au même titre qu'un parc de machines ou que les compétences des salariés 》) (p.4). Pour faire face à ce nouvel enjeu, les entreprises sont confrontées à la recherche de démarches spécifiques de gestion des connaissances, que l'on désigne le plus souvent sous le nom de knowledge management. La connaissance n'étant pas une ressource classique, elle entraîne de profonds bouleversements dans les logiques organisationnelles plus traditionnelles.
Dans le premier chapitre (pp. 9-2I), l'auteure tente de définir au plus près ce qu'est la connaissance organisationnelle qui est, pour certains, « une information à valeur ajoutée (par exemple un document scientifique ou technique) ; pour d'autres [... ] un savoir-faire, un tour de main propre à l'individu et difficilement explicitable; pour d'autres, enfin, $[. .$. une pratique de travail développée par un individu dans un poste donné » (p. 10). Si l'on considère différents travaux de référence, la connaissance serait d'abord un objet à situer par rapport aux données et aux informations. Pour d'autres chercheurs (Dzinkowski, «The Measurement and Management of Intellectual Capital : An Introduction », Management Accounting, vol. 78, 2000, pp. 32-26), elle serait un stock ou un patrimoine apparenté à des actifs de l'entreprise. A contrario, certains (Schubert et al., «A Global Knowledge Medium as a Virtual Community: The Netacademy Concept 》, pp. 618-620, in : Ellen D., Hoadley, Izak Benbasat, dirs, Proceedings of the Fourth Americas Conference on Information Systems, Baltimore, Association for Information Systems, 1998) y voient une condition d'accès à l'information et un processus permettant de savoir pour agir. D'autres (Jean-Marc Charlot, Agnès Lancini, « De la connaissance aux systèmes d'information supports », pp. |39-159, in : Frantz Rowe, dir., Faire de la recherche en systèmes d'information, Paris, Vuibert, 2002) font de la connaissance des individus un ressort de la productivité et de la compétitivité de l'entreprise. Des études (Chris Argyris, Donald Schön, Organizational Learning. A Theory of Action Perspective, Reading, Addison-Wesley, 1978) ont considéré la connaissance comme un processus organisationnel spécifique liant l'individu à l'organisation. Une perspective interactionniste (lkujiro Nonaka, Hirotaka Takeuchi, The Knowledge-Creating Company, New York, Oxford University Press, 1995) perçoit la connaissance comme le produit d'une interaction entre l'individu et l'organisation. Pour synthétiser ces différentes définitions, on considèrera que la connaissance est transmise entre individus ou de l'individu à l'organisation via l'information (soit gestuelle, soit orale, soit écrite). Dans le second chapitre de l'ouvrage (pp. 21-29), Aurélie Dudezert précise que, en matière de gestion des connaissances, les entreprises font souvent preuve d'un incroyable opportunisme managérial : « L'idée qui prévaut aujourd'hui est qu'il n'y a pas une "boîte à outils" de la gestion des connaissances qui permettrait de piloter la connaissance de manière optimale dans n'importe quelle organisation, mais qu'au contraire à chaque organisation correspond un mode de gestion des connaissances particulier » (p. 47). La gestion des 
connaissances étant une procédure de gestion très spécifique, elle demande à chaque entreprise une grande souplesse managériale et une grande capacité d'adaptation. Dans le troisième chapitre (pp. 30-38), l'auteure explique que les entreprises faisant le pari de centrer leurs activités autour de la connaissance sont obligées de réinventer de nouvelles modalités organisationnelles : « Le concept de l'entreprise centrée connaissance (knowledge-centric enterprise) aussi appelé organisation 2.0 s'est développé, [...] inspiré d'analyses théoriques de l'évolution de la gestion des connaissances et des organisations, mais aussi d'analyses de choix stratégiques et structurels de certaines entreprises »(p. 69). Ensuite, l'auteur prend l'exemple de la société Google qui, par son positionnement professionnel, valorise la connaissance de ses salariés qui sont tous, sans exception, considérés comme détenteurs de connaissances inestimables pour l'entreprise. Cela renforce encore la légende qui veut que, « chez Google, l'efficacité de l'entreprise repose sur l'ambition, la créativité, la liberté, le bien-être des salariés, la satisfaction du client et l'éthique » (p. 78). Ce sentiment d'appartenance est encouragé et recherché par les recruteurs de la société américaine qui vérifient scrupuleusement que leurs futurs salariés seront en totale adéquation avec le mythe et la culture Google.

Dans la conclusion de l'ouvrage (pp. 99-108), Aurélie Dudezert insiste sur le fait que l'émergence de la ressource connaissance dans les organisations - ressource qui n'est pas un phénomène naturel, mais un construit social - remet au cœur de l'organisation la question de l'action collective et la dimension sociale de la technologie. C'est là l'apport considérable de ce livre qui intéressera tous ceux (étudiants, futurs salariés, managers, chercheurs, etc.) qui s'intéressent de près aux mutations organisationnelles.

\section{Alexandre Eyries \\ 13M, université Nice Sophia Antipolis, F-06200 alex.eyries@yahoo.fr}

\section{François FARCY, Jean-François GAYRAUD, Le renseignement criminel.}

Paris, cnRs Éd., coll. Arès, 201 I, I 32 p.

$\|$ est des livres comme des régimes qualifiables d'« Ancien régime ». Celui de François Farcy et JeanFrançois Gayraud appartient à cette catégorie tant on comprend qu'il est spécialement écrit pour nourrir le corpus scientifique de la contestée $75^{\mathrm{e}}$ section du Conseil national des universités de « criminologie », créée le 13 février et supprimée le 6 août 2012. L'entreprise de légitimation est doublement signée : une publication dans la collection dirigée par Xavier Raufer et une préface rédigée par Alain Bauer (pp. 9-II). Ce dernier y vante ce « nouveau paradigme » (p. 10) que serait le renseignement, d'un point de vue analytique autant qu'opérationnel, pour la police. Depuis les séminaires de l'amiral Pierre Lacoste, au milieu des années 90, et notamment les constats de Jean-Marc Berlière (Le renseignement à la française, Paris, Éd. Economica, 1998, pp. 9-27, ici pp. 13-20), il est admis que la police, comme les douanes, participent du renseignement de sécurité et font preuve, autant que faire se peut, d'anticipation et de pro-activité.

Cela dit, le cours ouvrage des deux commissaires belge (François Farcy) et français (Jean-François Gayraud) se décline comme une dissertation d'étudiants... en « criminologie ».Après une longue introduction (pp. 1 3-24) destinée à présenter l'« approche géopolitique et globale des études criminelles » chère à Alain Bauer et Xavier Raufer (etYves Roucaute, «Une vocation nouvelle pour la criminologie », Sécurité globale, 5, automne 2008, pp.89-93, ici p. 9|), s'enchaînent deux chapitres déséquilibrés, présentant le renseignement et son acception criminelle - «Qu'est-ce que le renseignement criminel et à quoi sert-il ? » (pp. 25-64) - et expliquant comment en faire - « Comment fait-on du renseignement criminel » (pp. 65-93). Logiquement, une courte conclusion - « Contre la résilience criminelle, la profondeur du renseignement d'intérêt criminel » (pp. 95-96) - rappelant que le « renseignement inscrit l'activité policière dans le qualitatif et le moyen terme ("l'efficacité"), non dans le quantitatif et le court terme » (p. 95) vient clore la démonstration et ouvre sur une suite de quatre annexes présentant l'état actuel du renseignement criminel dans le monde anglo-saxon (pp. 99-102), en France (pp. 103109), en Belgique (pp. I | | - 125) et un cas pratique tiré de l'actualité des subprimes (pp. 127-128).

L'objectif affirmé du livre est de « proposer un nouvel outil » (p. 23) aux décideurs politiques et administratifs, à savoir le « renseignement criminel, une notion encore balbutiante » (p. 2l). Pour l'atteindre, les auteurs mobilisent les expériences anglo-saxonnes de community policing (« police de proximité ») et d'intelligence led policing (« fonction policière guidée par l'information $»)$, remontant aux années 70-80, mais surtout de l'expérience belge, élaborée empiriquement suite aux différentes affaires judiciaro-médiatiques (commandant François, 1980 ; Rebelle, 1992-1996 ; Dutroux, 1996-1998 ; Brabant Wallon, 1997) et une adaptation à l'environnement international (trafic de drogue, crimes organisés turc et kurde, terrorisme) (pp. 79-93). Toutefois, 